\title{
Integral Transforms and Their Applications to Scattering Theory
}

\author{
Jhasaketan Bhoi ${ }^{*}{ }^{*}$, Ujjwal Laha ${ }^{2}$ \\ 1, 2 Department of Physics, National Institute of Technology, Jamshedpur, Jharkhand-831014, India. \\ * Corresponding author. email: jskbhoi@gmail.com \\ Manuscript submitted July 12, 2014; accepted November 11, 2014. \\ doi: 10.17706/ijapm.2014.4.6.386-405
}

\begin{abstract}
Closed form analytical expressions for integral transforms of the free particle Green's functions by the form factors of the separable potential and Hankel function are evaluated by adopting various approaches to the problem to construct off-shell Jost/irregular solution of inhomogeneous Schrödinger type equation for motion in Graz separable potential. Off-shell Jost function is also computed numerically.
\end{abstract}

Key words: Green's functions, integral transforms, separable potential model, off-shell jost solution and function.

\section{Introduction}

Separable interactions have been frequently used in different area of physics such as particle, nuclear and atomic physics because of its simplicity involved in analytical calculation. In general non-local potential is a function of two coordinate variables. In the separable model $V_{\ell}\left(r, r^{\prime}\right)=\sum_{i=1}^{N} \lambda_{\ell}^{i}\left|g_{\ell}^{i}(r)\right\rangle\left\langle g_{\ell}^{i}\left(r^{\prime}\right)\right|$ with $\lambda_{\ell}^{i}$ and $g_{\ell}^{i}(r)$ represents the state dependent strength parameter and form factor of the potential. The attractive part of the nucleon-nucleon interaction involves a phenomenological intermediate region and a one pion exchange tail [1]. Therefore for a correct description of the nucleon-nucleon interaction in terms of the separable potential one needs at least two terms in the potential with the strength parameter having opposite signs. Since low energy scattering experiments sample out only the outer region of the potential, one term separable potential may be of importance for this energy range. For intermediate and high energy ranges one has to consider higher rank potential because of the sensitivity of scattering data to the choice of inner core irrespective of whether the separable potential is symmetric or non-symmetric [2]-[5], the associated Schrödinger equation can be solved in closed form.

The proton-proton and neutron-proton systems have been studied extensively with a large number of reliable experimental data [6]-[9]. They are rather accurate for proton-proton system while contain minor uncertainties for neutron-proton system [10]. By assuming charge symmetry which might be violated slightly [11] one can also extract information regarding neutron-neutron system from proton-proton observables with a proper treatment of the electromagnetic interaction [12], [13]. As a result, all realistic nucleon-nucleon interaction models exhibit similar on-shell properties despite the fact that they often result from different approaches to nucleon-nucleon dynamics [14], [15]. However, the situation is not so obvious with respect to the off-shell behaviour of the nucleon-nucleon interaction. The corresponding 
evidence can only be found in three or more particle problem involving nucleon-nucleon subsystems [16]. Though there exist numerous investigations of nucleon-nucleon off-shell feature over the last few decades or so, there is still much controversy and uncertainty about them [17]. Thus it is cleared how important it is to study off-shell feature of the nucleon-nucleon interaction. Most of the early separable models give poor fit to experimental data. An exception is the Graz separable potential [13], [18], [19] which produces reasonable fit to nucleon-nucleon observables. For inelastic scattering, however, one has to deal with the integral transforms of the free particle Green's function by the form factors of the separable potential and of the interacting Green's function by the Hankel function. The present text addresses itself to evaluate various integral transforms of the associated Green's functions and to construct exact analytical expressions for off-shell Jost solution for motion in Graz separable potential. In section 2 we develop various methods for construction of off-shell Jost solution for Graz potential in conjunction with integral transforms of the associated Green's functions. Section 3 is devoted for numerical results and discussions.

\section{Off-Shell Jost Solution}

The off-shell Jost solution $f_{\ell}^{S}(k, q, r)$ for Graz separable potential satisfies the inhomogeneous differential equation [20]-[22]

$$
\left[\frac{d^{2}}{d r^{2}}+k^{2}-\frac{\ell(\ell+1)}{r^{2}}\right] f_{\ell}^{S}(k, q, r)=d_{\ell}^{S}\left(\beta_{\ell}, k, q\right) g_{\ell}\left(\beta_{\ell}, r\right)+\left(k^{2}-q^{2}\right) e^{i \ell \pi / 2} \hat{h}_{\ell}^{(+)}(q r)
$$

with

$$
d_{\ell}^{S}\left(\beta_{\ell}, k, q\right)=\lambda_{\ell} \int_{0}^{\infty} d s g_{\ell}\left(\beta_{\ell}, s\right) f_{\ell}^{S}(k, q, s)
$$

and

$$
\hat{h}_{\ell}^{(+)}(x)=x h_{\ell}^{(+)}(x)=\sum_{L=0}^{\ell} \frac{(i)^{2 L-\ell}(\ell+L) !}{(2 i x)^{L} L !(\ell-L) !} e^{i x}
$$

Here $g_{\ell}\left(\beta_{\ell}, r\right)$ is the form factor of the Graz separable potential [18] written as

$$
g_{\ell}\left(\beta_{\ell}, r\right)=2^{-\ell}(\ell !)^{-1} r^{\ell} e^{-\beta_{\ell} r}
$$

The particular integral of (1) represents the off-shell Jost solution

$$
\begin{aligned}
f_{\ell}^{S}(k, q, r)= & \left(k^{2}-q^{2}\right) e^{i \ell \pi / 2} \int_{r}^{\infty} G_{\ell}^{0(I)}\left(r, r^{\prime}\right) \hat{h}_{\ell}^{(+)}\left(q r^{\prime}\right) d r^{\prime} \\
& +d_{\ell}^{S}\left(\beta_{\ell}, k, q\right) \int_{r}^{\infty} G_{\ell}^{0(I)}\left(r, r^{\prime}\right) \hat{h}_{\ell}^{(+)}\left(q r^{\prime}\right) d r^{\prime}
\end{aligned}
$$

where $G_{\ell}^{0(I)}\left(r, r^{\prime}\right)$, the irregular free particle Green's function written as 


$$
\left.G_{\ell}^{0(I)}\left(r, r^{\prime}\right)=-\frac{1}{\mathfrak{J}_{\ell}^{0}(k)}\left[\phi_{\ell}^{0}(k, r) f_{\ell}^{0}\left(k, r^{\prime}\right)-\phi_{\ell}^{0}\left(k, r^{\prime}\right) f_{\ell}^{0}(k, r)\right] \quad \text { for } r^{\prime}\right\rangle r
$$

with

$$
\begin{gathered}
\phi_{\ell}^{0}(k, r)=r^{\ell+1} e^{i k r} \Phi(\ell+1,2 \ell+2,-2 i k r), \\
f_{\ell}^{0}(k, r)=-(2 k r)^{\ell+1} i e^{i(k r-\ell \pi / 2)} \Psi(\ell+1,2 \ell+2,-2 i k r)
\end{gathered}
$$

and

$$
\mathfrak{J}_{\ell}^{0}(k)=\frac{(2 \ell+1) ! !}{k^{\ell}} e^{i \ell \pi / 2} f_{\ell}^{0}(k)=(2 k)^{-\ell} e^{i \ell \pi / 2} \frac{\Gamma(2 \ell+2)}{\Gamma(\ell+1)} .
$$

Expression in (5) involves some typical indefinite integrals. To circumvent the difficulties in evaluating such type of indefinite integrals we take recourse to different approaches to the problem to find closed form expression for the Jost solution for motion in the potential under consideration. The first step is to solve the inhomogeneous differential equation in (1) directly by applying certain transformations in conjunction with certain properties of special functions of mathematics.

\subsection{Off-Shell Jost Solution-Differential Equation Approach}

Transforming the dependent and independent variables in (1) by

$$
f_{\ell}^{S}(k, q, r)=r^{\ell+1} e^{i k r} F_{\ell}^{S}(k, q, r) \text { and } z=-2 i k r
$$

One has

$$
\begin{array}{r}
{\left[z \frac{d^{2}}{d z^{2}}+(2 \ell+2-z) \frac{d}{d z}-(\ell+1)\right] F_{\ell}^{S}(k, q, z)=-\frac{1}{2 i k} \sum_{n=0}^{\infty}\left[\frac{d_{\ell}^{S}\left(\beta_{\ell}, k, q\right) \gamma^{n}}{2^{\ell} \ell ! n !} z^{n}\right.} \\
\left.+\left(k^{2}-q^{2}\right)(-2 i k)^{\ell} \sum_{L=0}^{\ell} \frac{(i)^{2 L}(\ell+L) !}{L !(\ell-L) !}\left(-\frac{k}{q}\right)^{L} z^{n-\ell-L} \frac{\rho^{n}}{n !}\right]
\end{array}
$$

with $\gamma=\left(\beta_{\ell}+i k\right) / 2 i k$ and $\rho=(k-q) / 2 k$. Thus, in view of (10) and (11) the general solution [23] of (1) is written as

$$
\begin{aligned}
& f_{\ell}^{S}(k, q, r)=\left\{A_{1} \Phi(\ell+1,2 \ell+2 ;-2 i k r)+A_{2} \Psi(\ell+1,2 \ell+2 ;-2 i k r)\right. \\
& \quad-\frac{1}{2 i k} \sum_{n=0}^{\infty}\left[\frac{d_{\ell}^{S}\left(\beta_{\ell}, k, q\right)}{2^{\ell} \ell !} \frac{\gamma^{n}}{n !} \theta_{n+1}(\ell+1,2 \ell+2 ;-2 i k r)\right. \\
& \left.\left.+\left(k^{2}-q^{2}\right)(-2 i k)^{\ell} C_{L}(k, q) \frac{\rho^{n}}{n !} \theta_{n+1-\ell-L}(\ell+1,2 \ell+2 ;-2 i k)\right]\right\} r^{\ell+1} e^{i k}
\end{aligned}
$$


Here $\Phi(a, c ; z)$ and $\Psi(a, c ; z)$ are regular and irregular confluent hypergeometric functions [24], [25]. The other quantities $A_{1}$ and $A_{2}$ are two arbitrary constants and will be determined from the boundary conditions on $f_{\ell}^{S}(k, q, r)$. The factor $C_{L}(k, q)$ is defined as

$$
C_{L}(k, q)=\sum_{L=0}^{\ell} \frac{(i)^{2 L}(\ell+L) !}{L !(\ell-L) !}\left(-\frac{k}{q}\right)^{L}
$$

The off-shell Jost function satisfies the following boundary conditions

$$
f_{\ell}(k, q)=\operatorname{Lim}_{r \rightarrow 0}(q r)^{\ell} \frac{e^{-i \ell \pi / 2}}{(2 \ell+1) ! !} f_{\ell}(k, q, r) .
$$

and

$$
f_{\ell}(k, q, r) \underset{r \rightarrow \infty}{\longrightarrow} e^{i q r}
$$

Use of boundary condition at $r=0$ in (12) together with (14) yields

$$
A_{2}=G_{B}^{S}(k, q) f_{\ell}^{S}(k, q)
$$

with

$$
G_{B}^{S}(k, q)=\frac{e^{i \ell \pi / 2}(-2 i k)^{2 \ell+1} \Gamma(\ell+1)(2 \ell+1) ! !}{q^{\ell} \Gamma(2 \ell+2)} .
$$

The off-shell Jost function for Graz separable potential [21], [22] is given by

$$
f_{\ell}^{S}(k, q)=f_{\ell}^{0}(k, q)+\lambda_{\ell} \frac{\left(k^{2}-q^{2}\right) q^{\ell}}{(2 \ell+1) ! !} Y_{\ell}^{S}(k) \sum_{L=0}^{\ell} \frac{(i)^{L-\ell}(\ell+L) !}{D_{\ell}^{S}(k)(2 q)^{L} L !(\ell-L) !} Z_{\ell}^{S}\left(\beta_{\ell}, k, q\right)
$$

where $f_{\ell}^{0}(k, q)$ is the free-particle off-shell Jost function reads as

$$
\begin{array}{r}
f_{\ell}^{0}(k, q)=\frac{1}{(2 \ell+1) ! !}\left(\frac{q}{k+q}\right)^{\ell} \sum_{L=0}^{\ell} \frac{(\ell+1-L)(\ell+L) !}{L !}\left(\frac{q-k}{2 q}\right)^{L} \\
\times{ }_{2} F_{1}\left(\ell+1, \ell+L ; 2 \ell+2 ; \frac{2 k}{(k+q)}\right)
\end{array}
$$

and the other quantities $Y_{\ell}^{S}(k)$, and $Z_{\ell}^{S}\left(\beta_{\ell}, k, q\right)$ are 


$$
Y_{\ell}^{S}(k)=\int_{0}^{\infty} d r r^{\ell+1} e^{i k r} g_{\ell}\left(\beta_{\ell}, r\right) \Phi(\ell+1,2 \ell+2 ;-2 i k r)=\frac{\Gamma(2 \ell+2)}{2^{\ell} \ell !\left(\beta_{\ell}^{2}+k^{2}\right)^{\ell+1}},
$$

and

$$
Z_{\ell}^{S}\left(\beta_{\ell}, k, q\right)=-\frac{(i)^{L-\ell-1}}{2 i k 2^{\ell} \ell !} \frac{\partial^{\ell+1-L}}{\partial q^{\ell+1-L}} \sum_{n=0}^{\infty} \frac{\gamma^{n}}{n !} \operatorname{Lim}_{\varepsilon \rightarrow 0} \int_{0}^{\infty} d r e^{-(\varepsilon-i(k+q)) r} \theta_{n+1}(\ell+1,2 \ell+2 ;-2 i k r)
$$

Using the standard integral [23]

$$
\begin{array}{r}
\int_{0}^{\infty} d z e^{-b z} \theta_{\sigma}(a, c ; p z)=\frac{\Gamma(\sigma) p^{\sigma}}{(\sigma+c-1) b^{\sigma+1} 2} F_{1}(1, \sigma+a ; \sigma+c ; p / b), \\
\operatorname{Re} \sigma>0, \operatorname{Re}(\sigma+c)>1, \operatorname{Re} b>\operatorname{Re} p
\end{array}
$$

in above equation one obtains

$$
\begin{aligned}
& I_{z \ell}(k, q)=\sum_{n=0}^{\infty} \frac{\gamma^{n}}{n !} \operatorname{Lim}_{\varepsilon \rightarrow 0} \int_{0}^{\infty} d r e^{-(\varepsilon-i(k+q)) r} \theta_{n+1}(\ell+1,2 \ell+2 ;-2 i k r) \\
& \quad=\frac{2 i k}{(k+q)^{2}} \sum_{n=0}^{\infty}\left(\frac{k-i \beta_{\ell}}{k+q}\right)^{n} \frac{1}{(2 \ell+2+n)}{ }_{2} F_{1}\left(1, n+\ell+2 ; n+2 \ell+3 ; \frac{2 k}{k+q}\right)
\end{aligned}
$$

With the help of transformation relations [26]-[28]

$$
\begin{aligned}
&{ }_{2} F_{1}(a, b ; c ; z)= \frac{\Gamma(c) \Gamma(c-a-b)}{\Gamma(c-a) \Gamma(c-b)}{ }_{2} F_{1}(a, b ; a+b-c+1 ; 1-z) \\
&+(1-z)^{c-a-b} \frac{\Gamma(c) \Gamma(a+b-c)}{\Gamma(a) \Gamma(b)}{ }_{2} F_{1}(c-a, c-b ; c-a-b+1 ; 1-z) \\
&{ }_{2} F_{1}(a, b ; c ; z)=(1-z)^{-a}{ }_{2} F_{1}\left(a, c-b ; c ; \frac{z}{z-1}\right)
\end{aligned},
$$

and the integral representation of Gaussian hypergeometric function [26]-[28]

$$
{ }_{2} F_{1}(a, b ; c ; z)=\frac{\Gamma(c)}{\Gamma(b) \Gamma(c-b)} \int_{0}^{1} d t t^{b-1}(1-t)^{c-b-1}(1-t z)^{-a}
$$

we arrive at 


$$
\begin{aligned}
I_{z \ell}^{S}(k, q)= & \frac{\Gamma(-\ell) \Gamma(2 \ell+2)\left(q^{2}-k^{2}\right)^{\ell}}{(2 k)^{2 \ell} \Gamma(\ell+2)\left(\beta_{\ell}-i k\right)}{ }_{2} F_{1}\left(1,-\ell ; \ell+2 ; \frac{\beta_{\ell}+i k}{\beta_{\ell}-i k}\right) \\
& -\frac{\Gamma(2 \ell+2)}{\left(\beta_{\ell}-i k\right)}\left(\frac{q+k}{2 k}\right)^{2 \ell} \sum_{n=0}^{2 \ell+1} \frac{(-1)^{n}}{(n-\ell) n ! \Gamma(2 \ell+2-n)}\left(\frac{q-k}{q+k}\right)^{n} . \\
& \times{ }_{2} F_{1}\left(1, n-\ell ; n-\ell+1 ; \frac{(q-k)\left(\beta_{\ell}+i k\right)}{(q+k)\left(\beta_{\ell}-i k\right)}\right)
\end{aligned}
$$

Using the following three term recurrence relation [26]-[28]

$$
c_{2} F_{1}(a, b ; c ; z)-c_{2} F_{1}(a+1, b ; c ; z)+b z_{2} F_{1}(a+1, b+1 ; c+1 ; z)=0
$$

iteratively in the above equation $Z_{\ell}^{S}\left(\beta_{\ell}, k, q\right)$ is expressed as

$$
\begin{aligned}
& Z_{\ell}^{S}\left(\beta_{\ell}, k, q\right)=\frac{(i)^{L-\ell} \Gamma(2 \ell+2)}{2^{\ell} \ell !(2 k)^{2 \ell+1}} \frac{\partial^{\ell+1-L}}{\partial q^{\ell+1-L}}\left\{\frac { 1 } { \beta _ { \ell } - i k } \left[\frac{\Gamma(-\ell)}{\Gamma(\ell+2)}\left(q^{2}-k^{2}\right)^{\ell}\right.\right. \\
& { }_{2} F_{1}\left(1,-\ell ; \ell+2 ; \frac{\beta_{\ell}+i k}{\beta_{\ell}-i k}\right)-\frac{(q+k)^{2 \ell}}{(-\ell) \Gamma(2 \ell+2)}\left(\frac{2 i k}{\beta_{\ell}+i k}\right)^{2 \ell+1} \\
& \left.\left.\left\{{ }_{2} F_{1}\left(1,-\ell ;-\ell+1 ; \frac{(q-k)\left(\beta_{\ell}+i k\right)}{(q+k)\left(\beta_{\ell}-i k\right)}\right)-1\right\}-(q+k)^{2 \ell} X_{\ell}^{S}\left(\beta_{\ell}, k, q\right)\right]\right\}
\end{aligned}
$$

with

$$
\begin{aligned}
X_{\ell}^{S}\left(\beta_{\ell}, k, q\right)= & \frac{1}{(-\ell) \Gamma(2 \ell+2)}-\frac{(q-k)\left(\beta_{\ell}-i k\right)}{(q+k)\left(\beta_{\ell}+i k\right)(-\ell+1)} \sum_{n=0}^{2 \ell-1} \frac{(-1)^{n}}{\Gamma(n+3) \Gamma(2 \ell-n)} \\
& \times\left(\frac{\beta_{\ell}-i k}{\beta_{\ell}+i k}\right)^{n}\left({ }_{2} F_{1}\right)_{n+1}\left(1,-\ell+1 ;-\ell+2 ; \frac{(q-k)\left(\beta_{\ell}+i k\right)}{(q+k)\left(\beta_{\ell}-i k\right)}\right)
\end{aligned}
$$

Here $\left({ }_{2} F_{1}\right)_{n+1}(*)$, the first $(n+1)$ terms of the hypergeometric series with the given parameters and $D_{\ell}^{S}(k)$, the Fredholm determinant associated with regular/irregular boundary conditions [21], [29]

$$
\begin{aligned}
D_{\ell}^{S}(k)= & 1-\lambda_{\ell} \int_{0}^{\infty} \int_{0}^{r} d r d r^{\prime} g_{\ell}\left(\beta_{\ell}, r\right) G_{\ell}^{0(R)}\left(r, r^{\prime}\right) g_{\ell}\left(\beta_{\ell}, r^{\prime}\right) \\
= & 1-\frac{\lambda_{\ell} 2^{-2 \ell}(\ell !)^{-2} \Gamma(2 \ell+2)}{(\ell+1)\left(\beta_{\ell}-i k\right)}\left[\left(\beta_{\ell}^{2}+k^{2}\right)^{-\ell-1}{ }_{2} F_{1}\left(1,-\ell ; \ell+2 ; \frac{\left(\beta_{\ell}+i k\right)}{\left(\beta_{\ell}-i k\right)}\right) .\right. \\
& \left.-\frac{\left(2 \beta_{\ell}\right)^{-2 \ell-1}}{\left(\beta_{\ell}-i k\right)}{ }_{2} F_{1}\left(1,-\ell ; \ell+2 ;\left(\frac{\left(\beta_{\ell}+i k\right)}{\left(\beta_{\ell}-i k\right)}\right)^{2}\right)\right]
\end{aligned}
$$


In view of (16) and (17) the Graz off-shell Jost solution reads as

$$
\begin{aligned}
f_{\ell}^{S}(k, q, r)= & \left\{A_{1} \Phi(\ell+1,2 \ell+2 ;-2 i k r)+G_{B}^{S}(k, q) f_{\ell}^{S}(k, q) \Psi(\ell+1,2 \ell+2 ;-2 i k r)\right. \\
& -\frac{1}{2 i k} \sum_{n=0}^{\infty}\left[\frac{d_{\ell}^{S}\left(\beta_{\ell}, k, q\right)}{2^{\ell} \ell !} \frac{\gamma^{n}}{n !} \theta_{n+1}(\ell+1,2 \ell+2 ;-2 i k r)+\left(k^{2}-q^{2}\right)\right. \\
& \left.\left.\times(-2 i k)^{\ell} C_{L}(k, q) \frac{\rho^{n}}{n !} \theta_{n+1-\ell-L}(\ell+1,2 \ell+2 ;-2 i k r)\right]\right\} r^{\ell+1} e^{i k r}
\end{aligned}
$$

The last two terms in (32), however, can be expressed in terms of indefinite integrals involving free particle regular Green's function [30]. The free particle regular Green's function [21], [30] is given by

$$
G_{\ell}^{0(R)}\left(r, r^{\prime}\right)=\frac{1}{\mathfrak{J}_{\ell}^{0}(k)}\left[\phi_{\ell}^{0}(k, r) f_{\ell}^{0}\left(k, r^{\prime}\right)-\phi_{\ell}^{0}\left(k, r^{\prime}\right) f_{\ell}^{0}(k, r)\right], \quad \text { for } \quad r^{\prime}\langle r
$$

Thus, in conjunction with (33) the last two terms of (32) read as

$$
-\frac{r^{\ell+1} e^{i k r}}{2 i k 2^{\ell} \ell !} \sum_{n=0}^{\infty} \frac{\gamma^{n}}{n !} \theta_{n+1}(\ell+1,2 \ell+2 ;-2 i k r)=\int_{0}^{r} G_{\ell}^{0 R)}\left(r, r^{\prime}\right) g_{\ell}\left(\beta_{\ell}, r^{\prime}\right) d r^{\prime}
$$

and

$$
\begin{gathered}
\left(k^{2}-q^{2}\right)(-2 i k)^{\ell-1} C_{L}(k, q) r^{\ell+1} e^{i k r} \sum_{n=0}^{\infty} \frac{\rho^{n}}{n !} \theta_{n+1-\ell-L}(\ell+1,2 \ell+2 ;-2 i k r) \\
=e^{i \ell \pi / 2} \int_{0}^{r} G_{\ell}^{0(R)}\left(r, r^{\prime}\right)\left(k^{2}-q^{2}\right) \hat{h}_{\ell}^{(+)}\left(q r^{\prime}\right) d r^{\prime}
\end{gathered}
$$

To calculate the quantity $d_{\ell}^{S}\left(\beta_{\ell}, k, q\right)$ defined in (2) we proceed as follows.

Multiplying (5) by $\lambda_{\ell} g_{\ell}\left(\beta_{\ell}, r\right)$ on both sides and integrating over the whole range one has

$$
\begin{aligned}
d_{\ell}^{S}\left(\beta_{\ell}, k, q\right) & =\left(k^{2}-q^{2}\right) \frac{\lambda_{\ell} e^{i \ell \pi / 2}}{D_{\ell}^{S}(k)} \int_{0}^{\infty} \int_{r}^{\infty} d r d r^{\prime} g_{\ell}\left(\beta_{\ell}, r\right) G_{\ell}^{0(I)}\left(r, r^{\prime}\right) \hat{h}_{\ell}^{(+)}\left(q r^{\prime}\right) \\
& =\frac{\lambda_{\ell} e^{i \ell \pi / 2}}{D_{\ell}^{S}(k)}\left(k^{2}-q^{2}\right) \sum_{L=0}^{\ell} \frac{(i)^{L-\ell}(\ell+L) !}{(2 q)^{L} L !(\ell-L) !} Z_{\ell}^{S}\left(\beta_{\ell}, k, q\right)
\end{aligned} .
$$

In deriving the above result we have made use of (22) along with the following relation [23]

$$
\begin{aligned}
\theta_{\sigma}(a, c ; z) & =\frac{1}{(c-1)}\left[\Phi(a, c ; z) \int_{0}^{z} e^{-z^{\prime}} z^{\prime \sigma+c-2} \bar{\Phi}\left(a, c ; z^{\prime}\right) d z^{\prime}-\bar{\Phi}(a, c ; z) \int_{0}^{z} e^{-z^{\prime}} z^{\prime \sigma+c-2} \Phi\left(a, c ; z^{\prime}\right) d z^{\prime}\right] \\
& =\frac{z^{\sigma}}{\sigma(\sigma+c-1)}{ }_{2} F_{2}(1, \sigma+a ; \sigma+1, \sigma+c ; z) .
\end{aligned}
$$


Combination of (5), (6) and (31) yields

$$
\begin{aligned}
& M_{1 \ell}^{S}\left(\beta_{\ell}, k, q\right) r^{\ell+1} e^{i k r} \Phi(\ell+1,2 \ell+2 ;-2 i k r) \\
& \quad+M_{2 \ell}^{S}\left(\beta_{\ell}, k, q\right) r^{\ell+1} e^{i k r} \Psi(\ell+1,2 \ell+2 ;-2 i k r)=0
\end{aligned}
$$

with

$$
\begin{aligned}
M_{1 \ell}^{S}\left(\beta_{\ell}, k, q\right)= & A_{1}+\frac{d_{\ell}^{S}\left(\beta_{\ell}, k, q\right)}{\mathfrak{J}_{\ell}^{0}(k)} \int_{0}^{\infty} d r^{\prime} g_{\ell}\left(\beta_{\ell}, r^{\prime}\right) f_{\ell}^{0}\left(k, r^{\prime}\right) \\
& +\frac{e^{i \ell \pi / 2}\left(k^{2}-q^{2}\right)}{\mathfrak{J}_{\ell}^{0}(k)} \int_{0}^{\infty} d r^{\prime} \hat{h}_{\ell}^{(+)}\left(q r^{\prime}\right) f_{\ell}^{0}\left(k, r^{\prime}\right)
\end{aligned}
$$

and

$$
\begin{aligned}
M_{2 \ell}^{S}\left(\beta_{\ell}, k, q\right) & =C_{B}^{S}(k, q) f_{\ell}^{S}(k, q)-\frac{d_{\ell}^{S}\left(\beta_{\ell}, k, q\right) e^{-i(\ell+1) \pi / 2}}{(2 k)^{-\ell-1} \mathfrak{J}_{\ell}^{o}(k)} \\
& \times \int_{0}^{\infty} d r^{\prime} g_{\ell}\left(\beta_{\ell}, r^{\prime}\right) \phi_{\ell}^{0}\left(k, r^{\prime}\right)+\frac{e^{i \pi / 2}\left(k^{2}-q^{2}\right)}{(2 k)^{-\ell-1} \mathfrak{J}_{\ell}^{o}(k)} \int_{0}^{\infty} d r^{\prime} \hat{h}_{\ell}^{(+)}\left(q r^{\prime}\right) \phi_{\ell}^{0}\left(k, r^{\prime}\right)
\end{aligned}
$$

In view of (17)-(19), (36) and the standard relations

$$
\int_{0}^{\infty} e^{-\lambda z} z^{v} \Phi(a, c ; p z)=\frac{\Gamma(v+1)}{\lambda^{v+1}}{ }_{2} F_{1}(a, v+1 ; c ; p / \lambda)
$$

and

$$
f_{\ell}^{S}(k, q)=\frac{\left(k^{2}-q^{2}\right) q^{\ell}}{(2 \ell+1) ! !} \int_{0}^{\infty} d r \hat{h}_{\ell}^{(+)}(q r) \varphi_{\ell}(k, r)
$$

The above coefficient $M_{2 \ell}^{S}\left(\beta_{\ell}, k, q\right)$ in (40) becomes zero. Substituting (36) in (39) and evaluating the definite integrals with the help of the following relation [24], [25]

$$
\begin{gathered}
F\left(b, S ; 1+S+b-d ; 1-\frac{\mu}{a}\right)=\frac{a^{S} \Gamma(1+b+S-d)}{\Gamma(1+S-d) \Gamma(S)} \int_{0}^{\infty} e^{-a x} x^{S-1} \Psi(b, d ; \mu x) d x \\
\\
\operatorname{Re} S>0,1+\operatorname{Re} S>\operatorname{Re} d
\end{gathered} .
$$

We have

$$
\begin{aligned}
& A_{1}=-\frac{2^{-\ell}(\ell !)^{-1} d_{\ell}^{S}\left(\beta_{\ell}, k, q\right)}{(\ell+1)\left(\beta_{\ell}-i k\right)}{ }_{2} F_{1}\left(1,-\ell ; \ell+2 ; \frac{\beta_{\ell}+i k}{\beta_{\ell}-i k}\right)-(-1)^{\ell+1} \frac{e^{i(\ell+1) \pi / 2} \Gamma(\ell+1)}{(k+q)^{-\ell} \Gamma(2 \ell+2)} \\
& \times(q-k) \sum_{L=0}^{\ell} \frac{(\ell+L) !(\ell-L+1) \Gamma(1-\ell-L)}{L ! \Gamma(2-L)}\left(\frac{q+k}{2 q}\right)_{2}^{L} F_{1}\left(1-\ell-L,-\ell ; 2-L ; \frac{q-k}{q+k}\right) .
\end{aligned}
$$


Combination of (13), (16)-(19) along with (32), (36) and (44) gives the desired expression for off-shell Jost solution for Graz separable potential as

$$
\begin{aligned}
f_{\ell}^{S}(k, q, r) & =f_{\ell}^{0}(k, q, r)+\lambda_{\ell} \frac{e^{i \ell \pi / 2}\left(k^{2}-q^{2}\right)}{2^{\ell} \ell ! D_{\ell}^{S}(k)} \sum_{L=0}^{\ell} \frac{(i)^{L-\ell}(\ell+L) !}{(2 q)^{L} L !(\ell-L) !} \\
& \times Z_{\ell}^{S}\left(\beta_{\ell}, k, q\right)\left[\frac{-1}{\left(\beta_{\ell}-i k\right)(\ell+1)^{2}} F_{1}\left(1,-\ell ; \ell+2 ; \frac{\beta_{\ell}+i k}{\beta_{\ell}-i k}\right)\right. \\
& \times \Phi(\ell+1,2 \ell+2 ;-2 i k r)+\frac{(-2 i k)^{2 \ell+1} \Gamma(\ell+1)}{\left(\beta_{\ell}^{2}+k^{2}\right)^{\ell+1}} \\
& \left.\times \Psi(\ell+1,2 \ell+2 ;-2 i k r)-\frac{1}{2 i k} \sum_{n=0}^{\infty} \frac{\gamma^{n}}{n !} \theta_{n+1}(\ell+1,2 \ell+2 ;-2 i k r)\right] r^{\ell+1} e^{i k r}
\end{aligned}
$$

with

$$
\begin{aligned}
f_{\ell}^{0}(k, q, r) & =\sum_{L=0}^{\ell} \frac{e^{i \ell \pi / 2}(2 i k)^{2 \ell+1}(\ell+L) ! \Gamma(\ell+1)}{(2 q)^{L} L !(\ell-L) ! \Gamma(2 \ell+2)} r^{\ell+1} e^{i k r}\left\{\frac { \Gamma ( \ell - L + 2 ) } { ( k + q ) ^ { \ell - L } } \left[\frac{\Gamma(1-\ell-L)}{2 k \Gamma(2-L)}\right.\right. \\
& \times\left(\frac{q+k}{2 k}\right)^{\ell \ell}(q-k)_{2} F_{1}\left(1-\ell-L,-\ell ; 2-L ; \frac{q-k}{q+k}\right) \Phi(\ell+1,2 \ell+2 ;-2 i k r) \\
& \left.-\left(\frac{q-k}{q+k}\right)_{2}^{L} F_{1}\left(\ell+1, \ell+L ; 2 \ell+2 ; \frac{2 k}{q+k}\right) \Psi(\ell+1,2 \ell+2 ;-2 i k r)\right] \\
& \left.+\left(k^{2}-q^{2}\right) \frac{\Gamma(2 \ell+2)}{\Gamma(\ell+1)(2 k)^{\ell-L+2}} \sum_{n=0}^{\infty} \frac{\gamma^{n}}{n !} \theta_{n+1-\ell-L}(\ell+1,2 \ell+2 ;-2 i k r)\right\}
\end{aligned}
$$

\subsection{Off-Shell Jost Solution-Integral Transform of Green's Function}

The physical Green's function for Graz separable potential satisfies the inhomogeneous differential equation [21]

$$
\left[\frac{d^{2}}{d r^{2}}+k^{2}-\frac{\ell(\ell+1)}{r^{2}}\right] G_{\ell}^{S(+)}\left(r, r^{\prime}\right)=d_{\ell}^{S(+)}\left(\beta_{\ell}, k, r^{\prime}\right) g_{\ell}\left(\beta_{\ell}, r\right)+\delta\left(r-r^{\prime}\right)
$$

with

$$
d_{\ell}^{S(+)}\left(\beta_{\ell}, k, r^{\prime}\right)=\lambda_{\ell} \int_{0}^{\infty} d s g_{\ell}\left(\beta_{\ell}, s\right) G_{\ell}^{S(+)}\left(s, r^{\prime}\right)
$$

Let the function $F_{\ell}\left(r, r^{\prime}\right)$ be related to $G_{\ell}^{(+)}\left(r, r^{\prime}\right)$ by

$$
G_{\ell}^{S(+)}\left(r, r^{\prime}\right)=r^{\ell+1} e^{i k r} F_{\ell}^{S}\left(r, r^{\prime}\right)
$$


Then the integral transform $\bar{F}(r, q)=\left[F\left(r, r^{\prime}\right) ; r^{\prime} \rightarrow q\right]$ is related to $\bar{G}_{\ell}^{S(+)}(r, q)=\left[G_{\ell}^{S(+)}\left(r, r^{\prime}\right) ; r^{\prime} \rightarrow q\right]$ by

$$
\bar{G}_{\ell}^{S(+)}(r, q)=r^{\ell+1} e^{i k r} \bar{F}(r, q) .
$$

Equation (49) is inserted in (47) to get

$$
\begin{gathered}
{\left[r \frac{d^{2}}{d r^{2}}+(2 \ell+2+2 i k r) \frac{d}{d r}+2 i k(\ell+1)\right] F_{\ell}^{S}\left(r, r^{\prime}\right)=r^{-\ell} e^{-i k r} \delta\left(r-r^{\prime}\right)} \\
+\frac{d_{\ell}^{S(+)}\left(\beta_{\ell}, k, r^{\prime}\right)}{2^{\ell} \ell !} e^{-\left(\beta_{\ell}+i k\right) r}
\end{gathered}
$$

Taking the integral transform of (51) with respect to $\hat{h}_{\ell}^{(+)}\left(q r^{\prime}\right)\left[r^{\prime} \rightarrow q\right]$ and changing the independent variable by $z=-2 i k r$ one has

$$
\begin{aligned}
{\left[z \frac{d^{2}}{d z^{2}}+(2 \ell+\right.} & \left.2-z) \frac{d}{d z}+(\ell+1)\right] \bar{F}_{\ell}^{S}(z, q)=\sum_{L=0}^{\ell} \frac{(i)^{2 L-1}(-1)^{L+\ell-1}(\ell+L) !}{L !(\ell-L) ! 2^{-\ell+1} q^{L} k^{1-L-\ell}} \\
& \times \sum_{n=0}^{\infty} \frac{\rho^{n}}{n !} z^{n-\ell-L}-\frac{d_{\ell}^{S(+)}\left(\beta_{\ell}, k, q\right)}{2 i k 2^{\ell} \ell !} \sum_{n=0}^{\infty} \frac{\gamma^{n}}{n !} z^{n}
\end{aligned}
$$

The quantity $d_{\ell}^{S(+)}\left(\beta_{\ell}, k, q\right)$ is given by

$$
\begin{aligned}
d_{\ell}^{S(+)}\left(\beta_{\ell}, k, q\right) & =\int_{0}^{\infty} d r^{\prime} \hat{h}_{\ell}^{(+)}\left(q r^{\prime}\right) d_{\ell}^{S(+)}\left(\beta_{\ell}, k, r^{\prime}\right) \\
& =\lambda_{\ell} \int_{0}^{\infty} \int_{0}^{\infty} d r d r^{\prime} \hat{h}_{\ell}^{(+)}\left(q r^{\prime}\right) G_{\ell}^{S(+)}\left(r, r^{\prime}\right) g_{\ell}\left(\beta_{\ell}, r\right)
\end{aligned}
$$

Equation (52) represents a non-homogeneous confluent hypergeometric differential equation [23]. In view of (49) and (50), the complete primitive is

$$
\begin{aligned}
\bar{G}_{\ell}^{S(+)}(r, q) & =\left[B_{1} \Phi(\ell+1,2 \ell+2 ;-2 i k r)+B_{2} \Psi(\ell+1,2 \ell+2 ;-2 i k r)-\frac{(-2 k)^{\ell}}{2 i k}\right. \\
& \times \sum_{L=0}^{\ell} \frac{(i)^{2 L}(\ell+L) !}{L !(\ell-L) !}\left(-\frac{k}{q}\right)^{L} \Lambda_{\rho, \sigma}(\ell+1,2 \ell+2 ;-2 i k r)-\frac{d_{\ell}^{S(+)}\left(\beta_{\ell}, k, q\right)}{2 i k 2^{\ell} \ell !} \\
& \left.\times \Lambda_{\gamma, 1}(\ell+1,2 \ell+2 ;-2 i k r)\right] r^{\ell+1} e^{i k r}
\end{aligned}
$$

with $\sigma=1-\ell-L$. To determine the constants $B_{1}$ and $B_{2}$, boundary conditions on $\bar{G}_{\ell}^{S(+)}(r, q)$ will be applied judiciously at $r=0$ and $r \rightarrow \infty$. All the quantities in (54) except $\Psi(\ell+1,2 \ell+2 ;-2 i k r)$ are 
zero at $r=0$. Thus, one has $B_{2}=0$ and

$$
\begin{aligned}
& \bar{G}_{\ell}^{S(+)}(r, q)=\left\{B_{1} \Phi(\ell+1,2 \ell+2 ;-2 i k r)-\frac{1}{2 i k}\left[(-2 k)^{\ell} \sum_{L=0}^{\ell} \frac{(i)^{2 L}(\ell+L) !}{L !(\ell-L) !}\left(-\frac{k}{q}\right)^{L}\right.\right. \\
& \left.\left.\times \Lambda_{\rho, \sigma}(\ell+1,2 \ell+2 ;-2 i k r)+\frac{d_{\ell}^{S(+)}\left(\beta_{\ell}, k, q\right)}{2^{\ell} \ell !} \Lambda_{\gamma, 1}(\ell+1,2 \ell+2 ;-2 i k r)\right]\right\} r^{\ell+1} e^{i k r}
\end{aligned}
$$

To take the limit $r \rightarrow \infty$, the quantity $\bar{G}_{\ell}^{S(+)}(r, q)$ is expressed as

$$
\begin{aligned}
& \bar{G}_{\ell}^{S(+)}(r, q)=\int_{0}^{\infty} d r^{\prime} \hat{h}_{\ell}^{(+)}\left(q r^{\prime}\right) G_{\ell}^{S(+)}\left(r, r^{\prime}\right) \\
& =-k^{-1} e^{-i \ell \pi / 2}\left[f_{\ell}^{S}(k, r) \int_{0}^{r} d r^{\prime} \hat{h}_{\ell}^{(+)}\left(q r^{\prime}\right) \psi_{\ell}^{S(+)}\left(k, r^{\prime}\right)+\psi_{\ell}^{S(+)}(k, r) \int_{r}^{\infty} d r^{\prime} \hat{h}_{\ell}^{(+)}\left(q r^{\prime}\right) f_{\ell}^{S}\left(k, r^{\prime}\right)\right]
\end{aligned}
$$

where

$$
G_{\ell}^{S(+)}\left(r, r^{\prime}\right)=-k^{-1} e^{-i \ell \pi / 2} \psi_{\ell}^{S(+)}\left(k, r_{<}\right) f_{\ell}^{S}\left(k, r_{>}\right)
$$

Here $r_{<}$and $r_{>}$have their usual meaning. The functions $\psi_{\ell}^{S(+)}(k, r)$ and $f_{\ell}^{S}(k, r)$ stand for the on-shell physical and Jost solutions for Graz separable potential [18], [21], expressed as

$$
\psi_{\ell}^{S(+)}(k, r)=\psi_{\ell}^{0(+)}(k, r)+\frac{\lambda_{\ell}}{D_{\ell}^{S(+)}(k)} U_{\ell}^{S}\left(\beta_{\ell}, k\right) I_{\ell}^{S}\left(\beta_{\ell}, k, r\right)
$$

and

$$
f_{\ell}^{S}(k, r)=f_{\ell}^{0}(k, r)+\frac{\lambda_{\ell}}{D_{\ell}^{S}(k)} W_{\ell}^{S}\left(\beta_{\ell}, k\right) J_{\ell}^{S}\left(\beta_{\ell}, k, r\right)
$$

where

$$
\begin{gathered}
U_{\ell}^{S}\left(\beta_{\ell}, k\right)=\int_{0}^{\infty} d r g_{\ell}\left(\beta_{\ell}, r\right) \psi_{\ell}^{0(+)}(k, r)=\left[\frac{k^{\ell} \Gamma(\ell+1)}{(\ell !)\left(\beta_{\ell}{ }^{2}+k^{2}\right)^{\ell+1}}\right], \\
I_{\ell}^{S}\left(\beta_{\ell}, k, r\right)=\int_{0}^{\infty} d r^{\prime} g_{\ell}\left(\beta_{\ell}, r^{\prime}\right) G_{\ell}^{0(+)}\left(r, r^{\prime}\right)=-\frac{r^{\ell+1} e^{i k r}}{2 i k 2^{\ell} \ell !}\left[\frac{2 i k}{(\ell+1)\left(\beta_{\ell}-i k\right)} \times\right. \\
{ }_{2} F_{1}\left(1,-\ell ; \ell+2 ; \frac{\beta_{\ell}+i k}{\beta_{\ell}-i k}\right) \Phi(\ell+1,2 \ell+2 ;-2 i k r) \\
\left.+\sum_{n=0}^{\infty} \frac{\gamma^{n}}{n !} \theta_{n+1}(\ell+1,2 \ell+2 ;-2 i k r)\right]
\end{gathered}
$$




$$
\begin{aligned}
W_{\ell}^{S}\left(\beta_{\ell}, k\right) & =\int_{0}^{\infty} d r g_{\ell}\left(\beta_{\ell}, r\right) f_{\ell}^{0}(k, r) \\
& =\frac{e^{i \ell \pi / 2} \Gamma(2 \ell+2)}{2^{\ell+1} k^{\ell} \ell ! \Gamma(\ell+2)\left(\beta_{\ell}-i k\right)^{2}} F_{1}\left(1,-\ell ; \ell+2 ; \frac{\beta_{\ell}+i k}{\beta_{\ell}-i k}\right)
\end{aligned}
$$

and

$$
\left.J_{\ell}^{S}\left(\beta_{\ell}, k, r\right)=\int_{r}^{\infty} d r^{\prime} g_{\ell}\left(\beta_{\ell}, r^{\prime}\right) G_{\ell}^{0(I)}\left(r, r^{\prime}\right)=I_{\ell}^{S}\left(\beta_{\ell}, k, r\right)+f_{\ell}^{0}(k, r) U_{\ell}^{S} \beta_{\ell}, k\right)
$$

with $D_{\ell}^{S}(k)$, the Fredholm determinant associated with regular and irregular boundary conditions and $D_{\ell}^{S(+)}(k)$, with the physical boundary condition [21], [29] written as

$$
\begin{aligned}
D_{\ell}^{S(+)}(k) & =1-\lambda_{\ell} \int_{0}^{\infty} \int_{0}^{\infty} d r d r^{\prime} g_{\ell}\left(\beta_{\ell}, r\right) G_{\ell}^{0(+)}\left(r, r^{\prime}\right) g_{\ell}\left(\beta_{\ell}, r^{\prime}\right) \\
& =1+\lambda_{\ell}\left[\frac{\Gamma(2 \ell+2)\left(\beta_{\ell}-i k\right)^{-2}}{2^{2 \ell}(\ell !)^{2}\left(2 \beta_{\ell}\right)^{2 \ell+1}(\ell+1)}{ }_{2} F_{1}\left(1,-\ell ; \ell+2 ;\left(\frac{\beta_{\ell}+i k}{\beta_{\ell}-i k}\right)^{2}\right)\right]
\end{aligned}
$$

In Appendix-I we shall describe a different method to evaluate the double integral involved in (64).

Also, the last two terms in (55) can be expressed directly in terms of free particle regular Green's function as shown in (34) and (35). Substitution of (56)-(63) in (55) with the limit $r \rightarrow \infty$ one yields

$$
B_{1}=-\frac{1}{\mathfrak{J}_{\ell}^{0}(k)}\left\{\int_{0}^{\infty} d r \hat{h}_{\ell}^{(+)}(q r) f_{\ell}^{0}(k, r)+d_{\ell}^{S(+)}\left(\beta_{\ell}, k, q\right) \int_{0}^{\infty} d r g_{\ell}\left(\beta_{\ell}, r\right) f_{\ell}^{0}(k, r)\right\}
$$

and

$$
d_{\ell}^{S(+)}\left(\beta_{\ell}, k, q\right)=\frac{\lambda_{\ell}}{D_{\ell}^{S(+)}(k)} \int_{0}^{\infty} d r \hat{h}_{\ell}^{(+)}(q r) I_{\ell}^{S}\left(\beta_{\ell}, k, r\right)
$$

Evaluation of integrals in above equations leads to

$$
\begin{aligned}
B_{1} & =-\frac{e^{i \ell \pi / 2}}{\mathfrak{J}_{\ell}^{0}(k)(2 k)^{\ell}}\left[\frac{(-1)^{\ell} e^{i \pi / 2}}{(k+q)^{-\ell+1}} \sum_{L=0}^{\ell} \frac{(\ell+L) !(\ell-L+1) \Gamma(1-\ell-L)}{L ! \Gamma(2-L)}\left(\frac{q+k}{2 q}\right)^{L}\right. \\
& \times{ }_{2} F_{1}\left(1-\ell-L,-\ell ; 2-L ; \frac{q-k}{q+k}\right)+d_{\ell}^{S(+)}\left(\beta_{\ell}, k, q\right) \frac{2^{-\ell}(\ell !)^{-1} \Gamma(2 \ell+2)}{\Gamma(\ell+2)\left(\beta_{\ell}-i k\right)} \\
& \left.\times_{2} F_{1}\left(1,-\ell ; \ell+2 ; \frac{\beta_{\ell}+i k}{\beta_{\ell}-i k}\right)\right]
\end{aligned}
$$


with

$$
\begin{aligned}
& d_{\ell}^{S(+)}\left(\beta_{\ell}, k, q\right)=-\frac{\lambda_{\ell}}{2^{\ell}(\ell !) D_{\ell}^{S(+)}(k)}\left\{\frac{q^{-\ell} \Gamma(2 \ell+2)}{\left(k^{2}-q^{2}\right)\left(\beta_{\ell}-i k\right)} \frac{f_{\ell}^{0}(k, q)}{(\ell+1)} \times\right. \\
&\left.{ }_{2} F_{1}\left(1,-\ell ; \ell+2 ; \frac{\beta_{\ell}+i k}{\beta_{\ell}-i k}\right)-\sum_{L=0}^{\ell} \frac{(i)^{L-\ell}(\ell+L) !}{(2 q)^{L} L !(\ell-L) !} Z_{\ell}^{S}\left(\beta_{\ell}, k, q\right)\right\}
\end{aligned}
$$

The quantity $Z_{\ell}^{S}\left(\beta_{\ell}, k, q\right)$ has already been defined in (29). Combination of (55), (67) and (68) gives the desired expression for the integral transform of the Green's function for motion in Graz separable potential under consideration as

$$
\begin{aligned}
& \bar{G}_{\ell}^{S(+)}(r, q)=\bar{G}_{\ell}^{0(+)}(r, q)-d_{\ell}^{S(+)}\left(\beta_{\ell}, k, q\right) \times \\
&\left\{\frac{2^{-\ell}(\ell !)^{-1} e^{i \ell \pi / 2} \Gamma(2 \ell+2)}{\mathfrak{J}_{\ell}^{0}(k)(2 k)^{\ell} \Gamma(\ell+2)\left(\beta_{\ell}-i k\right)^{2}} F_{1}\left(1,-\ell ; \ell+2 ; \frac{\beta_{\ell}+i k}{\beta_{\ell}-i k}\right)\right. \\
&\left.\times \Phi(\ell+1,2 \ell+2 ;-2 i k r)+\frac{1}{2 i k 2^{\ell} \ell !} \Lambda_{\gamma, 1}(\ell+1,2 \ell+2 ;-2 i k r)\right\} r^{\ell+1} e^{i k r} .
\end{aligned}
$$

The expression for $\bar{G}_{\ell}^{S(+)}(r,-q)$ is obtained by replacing $q$ by $-q$ in above equation according to

$$
\bar{G}_{\ell}^{S(+)}(r,-q)=(-1)^{\ell} \int_{0}^{\infty} d r^{\prime} G_{\ell}^{S(+)}\left(r, r^{\prime}\right) \hat{h}_{\ell}^{(+)}\left(-q r^{\prime}\right)=(-1)^{\ell}\left|\bar{G}_{\ell}^{S(+)}(r, q)\right|_{q \rightarrow-q}
$$

The off-shell physical solution for the motion in Graz separable potential is obtained by using the following relation

$$
\psi_{\ell}^{S(+)}(k, q, r)=\frac{\left(k^{2}-q^{2}\right)}{2 i}\left[\bar{G}_{\ell}^{S(+)}(r, q)-(-1)^{\ell} \bar{G}_{\ell}^{S(+)}(r,-q)\right]
$$

Having the expression for off-shell physical solution one can identify the corresponding off-shell Jost solution by exploiting the following relation [21]

$$
\begin{aligned}
\psi_{\ell}^{(+)}(k, q, r)= & -\frac{\pi q}{2} T_{\ell}\left(k, q, k^{2}\right) e^{-i \ell \pi / 2} f_{\ell}(k, r) \\
& +\frac{1}{2 i}\left[e^{-i \ell \pi / 2} f_{\ell}(k, q, r)-e^{i \ell \pi / 2} f_{\ell}(k,-q, r)\right]
\end{aligned}
$$

with

$$
T_{\ell}\left(k, q, k^{2}\right)=\left(\frac{k}{q}\right)^{2} \frac{\left[f_{\ell}(k, q)-f_{\ell}(k,-q)\right]}{2 i f_{\ell}(k)}
$$


the half off-shell T-matrix. We have identified the off-shell Jost solution for motion in the Graz separable potential is in exact agreement with (45). In the following we shall discuss another approach to the problem.

\subsection{Off-Shell Jost Solution-Direct Integration Approach}

The particular integral of (1), represents the off-shell Jost solution [31], written as

$$
f_{\ell}^{S}(k, q, r)=\left(k^{2}-q^{2}\right) e^{i \ell \pi / 2} \int_{r}^{\infty} G_{\ell}^{S(I)}\left(r, r^{\prime}\right) \hat{h}_{\ell}^{(+)}\left(q r^{\prime}\right) d r^{\prime}
$$

Here $G_{\ell}^{S(I)}\left(r, r^{\prime}\right)$ is the irregular Green's function for motion in the potential under consideration and is rewritten in terms of free particle irregular Green's function and their integral transforms as

$$
G_{\ell}^{S(I)}\left(r, r^{\prime}\right)=G_{\ell}^{0(I)}\left(r, r^{\prime}\right)+\frac{\lambda_{\ell}}{D_{\ell}^{S}(k)} \bar{G}_{\ell}^{0(I)}\left(\beta_{\ell}, r^{\prime}\right) \int_{r}^{\infty} G_{\ell}^{0(I)}\left(r, r^{\prime}\right) g_{\ell}\left(\beta_{\ell}, r^{\prime}\right) d r^{\prime}
$$

with

$$
\bar{G}_{\ell}^{0(I)}\left(\beta_{\ell}, r^{\prime}\right)=\int_{0}^{\infty} G_{\ell}^{0(I)}\left(r, r^{\prime}\right) g_{\ell}\left(\beta_{\ell}, r\right) d r
$$

Substituting (75) in (74) one has

$$
\begin{aligned}
f_{\ell}^{S}(k, q, r)= & \left(k^{2}-q^{2}\right) e^{i \ell \pi / 2}\left[\int_{r}^{\infty} G_{\ell}^{0(I)}\left(r, r^{\prime}\right) \hat{h}_{\ell}^{(+)}\left(q r^{\prime}\right) d r^{\prime}+\frac{\lambda_{\ell} \bar{G}_{\ell}^{0(I)}\left(\beta_{\ell}, q\right)}{D_{\ell}^{S}(k)}\right. \\
& \left.\times \int_{r}^{\infty} G_{\ell}^{0(I)}\left(r, r^{\prime}\right) g_{\ell}\left(\beta_{\ell}, r^{\prime}\right) d r^{\prime}\right] \\
= & f_{\ell}^{0}(k, q, r)+\frac{\lambda_{\ell} e^{i \ell \pi / 2}}{D_{\ell}^{S}(k)}\left(k^{2}-q^{2}\right) \bar{G}_{\ell}^{0(I)}\left(\beta_{\ell}, q\right) \int_{r}^{\infty} G_{\ell}^{0(I)}\left(r, r^{\prime}\right) g_{\ell}\left(\beta_{\ell}, r^{\prime}\right) d r^{\prime}
\end{aligned}
$$

where

$$
\bar{G}_{\ell}^{0(I)}\left(\beta_{\ell}, q\right)=\int_{0}^{\infty} \int_{r}^{\infty} \hat{h}_{\ell}^{(+)}\left(q r^{\prime}\right) G_{\ell}^{0(I)}\left(r, r^{\prime}\right) g_{\ell}\left(\beta_{\ell}, r\right) d r d r^{\prime}
$$

The indefinite integral involved in (77) can be evaluated by rewriting it as

$$
\int_{r}^{\infty} G_{\ell}^{0(I)}\left(r, r^{\prime}\right) g_{\ell}\left(\beta_{\ell}, r^{\prime}\right) d r^{\prime}=\int_{0}^{\infty} G_{\ell}^{0(I)}\left(r, r^{\prime}\right) g_{\ell}\left(\beta_{\ell}, r^{\prime}\right) d r^{\prime}-\int_{0}^{r} G_{\ell}^{0(I)}\left(r, r^{\prime}\right) g_{\ell}\left(\beta_{\ell}, r^{\prime}\right) d r^{\prime} .
$$

The definite and indefinite integrals involved in (78) and (79) can easily be handled by substituting the free particle irregular Green's function [24], [25], [30] together with the relations (26), (37), (41), (43) 


$$
\Psi(a, b ; z)=\frac{\Gamma(1-b)}{\Gamma(a-b+1)} \Phi(a, b ; z)+\frac{\Gamma(b-1)}{\Gamma(a)} z^{1-b} \Phi(a-b+1,2-b ; z)
$$

and

$$
{ }_{2} F_{1}(a, b ; c ; z)=(1-z)^{c-a-b}{ }_{2} F_{1}(c-a, c-b ; c ; z) .
$$

All these expressions when substituted in (77) generate the desired expression of the off-shell Jost solution for the Graz separable potential [i.e. (45)].

\section{Numerical Results and Discussions}

For the s-wave case the form factor of the Graz-separable potential exactly coincides with that of Yamaguchi [2]. In the following we shall verify that for the s-wave case (45) exactly coincides with that of Yamaguchi off-shell Jost solution [22], [32]-[36]. For $\ell=0$, (45) becomes

$$
\begin{aligned}
f_{0}^{S}(k, q, r)= & f_{0}^{0}(k, q, r)+\lambda_{0} \frac{\left(k^{2}-q^{2}\right)}{D_{0}^{S}(k)} Z_{0}^{S}\left(\beta_{0}, k, q\right)\left[\frac{-1}{\left(\beta_{0}-i k\right)} \Phi(1,2 ;-2 i k r)\right. \\
& \left.+\frac{(-2 i k)}{\left(\beta_{0}^{2}+k^{2}\right)} \Psi(1,2 ;-2 i k r)-\frac{1}{2 i k} \sum_{n=0}^{\infty} \frac{\gamma^{n}}{n !} \theta_{n+1}(1,2 ;-2 i k r)\right] r e^{i k r}
\end{aligned}
$$

with the free particle s-wave Jost solution

$$
\begin{array}{r}
f_{0}^{0}(k, q, r)=2 i k r e^{i k r}\left[\frac{q-k}{2 k} \Phi(1,2 ;-2 i k r)-\Psi(1,2 ;-2 i k r)\right. \\
\left.+\frac{\left(k^{2}-q^{2}\right)}{(2 k)^{2}} \sum_{n=0}^{\infty} \frac{\rho^{n}}{n !} \theta_{n+1}(1,2 ;-2 i k r)\right]
\end{array}
$$

Using the integral representation of $\Phi(a, c ; z)$ and $\Psi(a, c ; z)$

$$
\Phi(a, c ; z)=\frac{\Gamma(c)}{\Gamma(a) \Gamma(c-a)} \int_{0}^{1} e^{z u} u^{a-1}(1-u)^{c-a-1} d u ; \operatorname{Re} c>\operatorname{Re} a>0
$$

and

$$
\Psi(a, c ; z)=\frac{1}{\Gamma(a)} \int_{0}^{\infty} e^{-z t} t^{a-1}(1+t)^{c-a-1} d t ; \operatorname{Re} a>o
$$

together with the relation

$$
\theta_{\sigma}(1, c ; z)=\frac{z^{\sigma}}{\sigma(\sigma+c-1)} \Phi(1, \sigma+c ; z)
$$


equations (82) leads to

$$
f_{0}^{S}(k, q, r)=e^{i q r}+\lambda_{0} \frac{\left(\beta_{0}+i q\right)}{D_{0}^{S}(k)\left(\beta_{0}^{2}+q^{2}\right)\left(\beta_{0}^{2}+k^{2}\right)} e^{-\beta_{0} r}
$$

with

$$
D_{0}^{S}(k)=1-\frac{\lambda_{0}}{2 \beta_{0}\left(\beta_{0}^{2}+k^{2}\right)} .
$$

The result in (87) is in exact agreement with that of Ghosh et al. [34]. Other useful checks on (45) consist in showing that $f_{\ell}^{S}(k, q, r) \underset{r \rightarrow 0}{\rightarrow} f_{\ell}^{S}(k, q)$ i.e. it reproduces (18); when $\lambda_{\ell}=0, f_{\ell}^{S}(k, q, r)$ goes to free particle one [i.e.(19)] and for $q=k$

$$
\begin{aligned}
f_{\ell}^{S}(k, r)=\left.f_{\ell}^{S}(k, q, r)\right|_{q \rightarrow k} & -(2 k r)^{\ell+1} i e^{i(k r-\ell \pi / 2)} \Psi(\ell+1,2 \ell+2 ;-2 i k r)-\frac{\lambda_{\ell} \Gamma(2 \ell+2) r^{\ell+1} e^{i(k r+\pi / 2)}}{D_{\ell}^{S}(k)(\ell !)^{2} \Gamma(\ell+2)\left(\beta_{\ell}-i k\right)} \times \\
& { }_{2} F_{1}\left(1,-\ell ; \ell+2 ; \frac{\beta_{\ell}+i k}{\beta_{\ell}-i k}\right)\left\{\frac{\Gamma(\ell+1) k^{\ell+1}}{\left(\beta_{\ell}^{2}+k^{2}\right)^{\ell+1}} \Psi(\ell+1,2 \ell+2,-2 i k r)\right. \\
& -\frac{e^{i \ell \pi / 2}}{k^{\ell+1} 2^{2 \ell+2}}\left[\frac{2 i k}{(\ell+1)\left(\beta_{\ell}-i k\right)}{ }_{2} F_{1}\left(1,-\ell ; \ell+2 ; \frac{\beta_{\ell}+i k}{\beta_{\ell}-i k}\right) \times\right. \\
& \left.\left.\Phi(\ell+1,2 \ell+2 ;-2 i k r)+\sum_{n=0}^{\infty} \frac{\gamma^{n}}{n !} \theta_{n+1}(\ell+1,2 \ell+2 ;-2 i k r)\right]\right\},
\end{aligned}
$$

the on-shell Jost solution. The off-shell Jost solution and Jost function are continuous functions of the off-shell momentum $q$ for Graz potential. It is well known that the phase of the Jost function is the negative of the scattering phase shift $\delta(k)$. Therefore, one gets the scattering phase shifts from the knowledge of the Jost function and one has

$$
\tan \delta(k)=-\operatorname{Im} f^{S}(k) / \operatorname{Re} f^{S}(k)
$$

Further, we define a quantity $\Delta(k, q)$ termed as quasi phase

$$
\tan \Delta(k, q)=-\operatorname{Im} f^{S}(k, q) / \operatorname{Re} f^{S}(k, q) .
$$

As $f^{S}(k, q)$ is a continuous function of $q$ equation (91) produces the phase-shift $\delta(k)$ when $q \rightarrow k$. In the following we portray the results of $\Delta(k, q)$ in Fig. 1 as a function of $q$ and verify that it produces the scattering phase shift [37] $\delta(k)$ at $q=k$ for scattering by Yamaguchi potential [2]. 


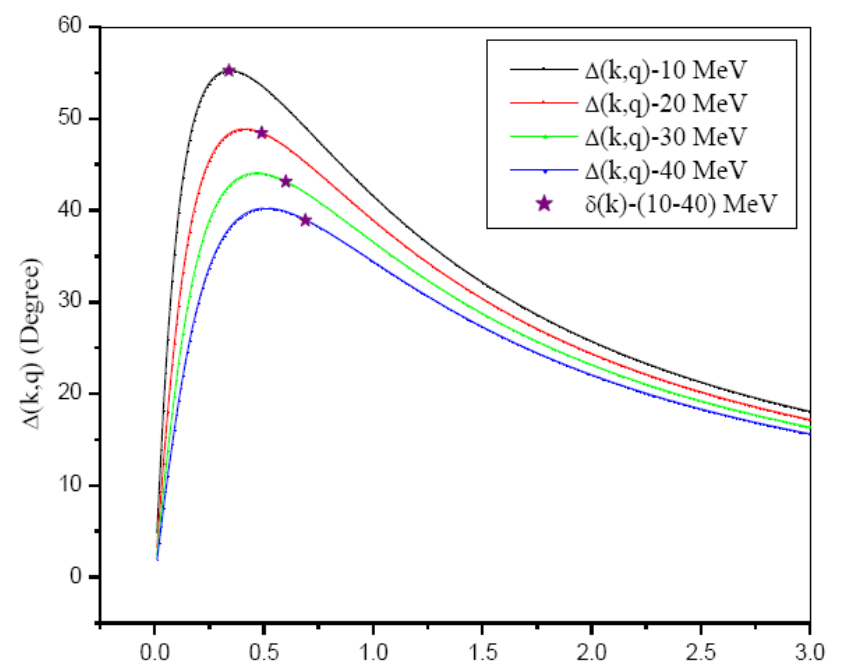

Fig. 1. $\Delta(k, q)$ as a function of off-shell momentum $q$.

Appendix

$$
\bar{G}_{\ell}^{0(+)}\left(\alpha_{\ell}, \beta_{\ell}\right)=\int_{0}^{\infty} \int_{0}^{\infty} d r d r^{\prime} g_{\ell}\left(\beta_{\ell}, r\right) G_{\ell}^{0(+)}\left(r, r^{\prime}\right) g_{\ell}\left(\beta_{\ell}, r^{\prime}\right)
$$

The partially projected Green's function satisfies the differential equation

$$
\left[\frac{\partial^{2}}{\partial r^{2}}-\frac{2 \ell}{r} \frac{\partial}{\partial r}+k^{2}\right] G_{\ell}^{0(+)}\left(r, r^{\prime}\right)=2\left(r r^{\prime}\right)^{\ell} \delta\left(r-r^{\prime}\right) .
$$

Taking the double Laplace transforms of equation (A1) with respect to $\alpha$ and $\beta$ we have

$$
\left[\left(\beta_{\ell}{ }^{2}+k^{2}\right) \frac{\partial}{\partial \alpha_{\ell}}+2(\ell+1) \alpha_{\ell}\right] \tilde{G}_{\ell}^{0(+)}\left(\alpha_{\ell}, \beta_{\ell}\right)=-\frac{2}{\left(\alpha_{\ell}+\beta_{\ell}\right)^{2 \ell+2}},
$$

where

$$
\tilde{G}_{\ell}^{0(+)}\left(\alpha_{\ell}, \beta_{\ell}\right)=\frac{\bar{G}_{\ell}^{0(+)}\left(\alpha_{\ell}, \beta_{\ell}\right)}{\Gamma(2 \ell+2)}=\frac{L_{2}\left[G_{\ell}^{0(+)}\left(r, r^{\prime}\right) ; \alpha_{\ell}, \beta_{\ell}\right]}{\Gamma(2 \ell+2)} .
$$

Using the following transformation

$$
\tilde{G}_{\ell}^{0(+)}\left(\alpha_{\ell}, \beta_{\ell}\right)=\frac{\chi^{2 \ell+2}}{\left(\alpha_{\ell}+\beta_{\ell}\right)^{2 \ell+2}} u(\chi) ; \chi=-\frac{2 i k\left(\alpha_{\ell}+\beta_{\ell}\right)}{\left(\alpha_{\ell}-i k\right)\left(\beta_{\ell}-i k\right)}
$$

in (A2) one obtains 


$$
\left((1-\chi) \frac{d}{d \chi}-(\ell+1)\right) u_{\ell}(\chi)=2(2 i k)^{2 \ell+1} \chi^{-(2 \ell+2)}
$$

Using another transformation

$$
u_{\ell}(\chi)=\chi^{-(2 \ell+1)} f_{\ell}(\chi) ; \xi=1-\chi
$$

and differentiating the resultant equation with respect to $\xi$ one gets

$$
\left[\xi(1-\xi) \frac{d^{2}}{d \xi^{2}}+\{(\ell+2)+(\ell-2) \xi\} \frac{d}{d \xi}+\ell\right] f_{\ell}(\xi)=0
$$

The solution of (A7) is well known and reads as

$$
f_{\ell}(\xi)=B_{2} F_{1}(1,-\ell ; \ell+2 ; \xi)
$$

where $B$ is an arbitrary constant and has to be determined from the boundary condition. In view of (A4), (A6) and (A8) we have

$$
\tilde{G}_{\ell}^{0(+)}\left(\alpha_{\ell}, \beta_{\ell}\right)=-B \frac{\left[(2 i k)\left(\alpha_{\ell}+\beta_{\ell}\right)\right]^{-(2 \ell+1)}}{\left(\alpha_{\ell}-i k\right)\left(\beta_{\ell}-i k\right)}{ }_{2} F_{1}\left(1,-\ell ; \ell+2 ; \frac{\left(\alpha_{\ell}+i k\right)\left(\beta_{\ell}+i k\right)}{\left(\alpha_{\ell}-i k\right)\left(\beta_{\ell}-i k\right)}\right) .
$$

From the behaviour of $\tilde{G}_{\ell}^{0(+)}\left(\alpha_{\ell}, \beta_{\ell}\right)$ for large values of $\alpha_{\ell}$ and $\beta_{\ell}$ we obtain

$$
B=-\frac{(2 i k)^{(2 \ell+1)}}{(\ell+1)}
$$

Combining (A3), (A9) and (A10) the double transform of free particle Green's function by the form factors of the Graz separable potential is expressed as

$$
\bar{G}_{\ell}^{0(+)}\left(\alpha_{\ell}, \beta_{\ell}\right)=-\frac{\Gamma(2 \ell+2)\left(\alpha_{\ell}+\beta_{\ell}\right)^{-(2 \ell+1)}}{(\ell+1)\left(\alpha_{\ell}-i k\right)\left(\beta_{\ell}-i k\right)}{ }_{2} F_{1}\left(1,-\ell ; \ell+2 ; \frac{\left(\alpha_{\ell}+i k\right)\left(\beta_{\ell}+i k\right)}{\left(\alpha_{\ell}-i k\right)\left(\beta_{\ell}-i k\right)}\right) .
$$

For $\alpha_{\ell}=\beta_{\ell}$ one gets the desired expression (64).

\section{References}

[1] Brown, G. E., \& Jackson, A. D. (1976). The Nucleon-Nucleon Interaction. Amsterdam: North-Holland Publishing Company.

[2] Yamaguchi, Y. (1954). Two-nucleon problem when the potential is nonlocal but separable. I. Phys. Rev., 95, 1628-1634. 
[3] Tabakin, F. (1968). Single separable potential with attraction and repulsion. Phys. Rev., 174, 1208-1212.

[4] Mongan, T. R. (1968). Separable-potential fits to nucleon-nucleon scattering data. Phys. Rev., 175, 1260-1274.

[5] Saito, S. (1968). Effect of pauli principle in scattering of two clusters progr. Theor. Phys., 40, 893-894.

[6] Arndt, R. A., Roper, L. D., Bryan, R. A., Clark, R. B., VerWest, B. J., \& Signell, P. (1983). Nucleon-nucleon partial-wave analysis to $1 \mathrm{GeV}$. Phys. Rev. D, 28, 97-122.

[7] Bystricki, J. et al. (1980). Landolt-Börnstein. Berlin: Springer.

[8] MacGregor, M. H., Arndt, R. A., \& Wright, R. M. (1969). Determination of the nucleon - nucleon scattering matrix. X. (p, p) and (n, p) analysis from 1 to 450 MeV. Phys. Rev., 182, 1714-1728.

[9] Wiringa, R. B., Stoks, V. G. J., \& Schiavilla, R. (1995). Accurate nucleon-nucleon potential with charge-independence breaking. Phys. Rev. C, 51, 38-51.

[10] Bugg, D. V. (1985). Nucleon-nucleon physics up to $1 \mathrm{GeV}$. Annual Review of Nuclear and Particle Science, $35,295-320$.

[11] Van, O. W. T. H. (1982). On the evidence of charge symmetry breaking. Comments Nucl. Part. Phys., 10, 251-262.

[12] Plessas, W., Mathelitsch, L., \& Pauss, F. (1981). Influence of the Coulomb-distortion effect on proton-proton observables. Phys. Rev. C, 23, 1340-1354.

[13] Schwinger, W., Plessas, W., Kok, L. P., \& Van, H. H. (1983). Separable representation of the nuclear proton-proton interaction. Phys. Rev. C, 27, 515-522.

[14] Haidenbauer, J., \& Plessas, W. (1983). Separable approximations of two-body interactions. Phys. Rev. C, 27, 63-70.

[15] Plessas, W. (1982). On-shell and off-shell behaviour of separable nucleon-nucleon potentials. Acta Phys. Austriaca, 54, 305-338.

[16] Fearing, H. W., Goldstein, G. R., \& Moravesik, M. J. (1984). Amplitude structure of off-shell processes. Phys. Rev. D, 29, 2612-2624.

[17] Thomas, A. W. (1978). Few-Body Systems and Nuclear Forces II. Berlin: Springer.

[18] Crepinsek, L., Lang, C. B., Oberhumer, H., Plessas, W., \& Zingl, H. (1975). A realistic separable nucleon-nucleon potential with regard to the coulomb interaction. Acta Phys. Austriaca, 42, 139-162.

[19] Zankel, H., Plessas, W., \& Haidenbauer, J. (1983). Sensitivity of N-d polarization observables on the off-shell behavior of the N-N interaction. Phys. Rev. C, 28, 538-541.

[20] Leeuwen, J. M. J., \& Reiner, A. S. (1961). On the calculation of the t-matrix for potentials with a hard core. Physica, 27, 99-110.

[21] Laha, U., \& Talukdar, B. (1991). Half-shell T-matrix for coulomb-modified Graz separable potential. Pramana-J. Phys., 36, 289-304.

[22] Laha, U. (2006). Off-shell jost solution for a coulomb-like potential. Phys. Rev. A, 74, 012710.

[23] Babister, A. W. (1967). Transcendental Functions Satisfying Non-Homogeneous Linear Differential Equations. New York: The MacMillan Company.

[24] Slater, L. J. (1960). Confluent Hypergeometric Functions. New York: Cambridge University Press.

[25] Buchholz, H. (1969). The Confluent Hypergeometric Function. New York: Springer.

[26] Erdeyli, A. (1953). Higher Transcendental Functions. New York: McGraw-Hill.

[27] Magnus, W., \& Oberhettinger, F. (1949). Formulas and Theorems for the Special Functions of Mathematical Physics. New York: Chelsea.

[28] Slater, L. J. (1966). Generalized Hypergeometric Functions. Cambridge: Cambridge University Press.

[29] Laha, U., Roy, B. J., \& Talukdar, B. (1889). Trasform of the coulomb green function by the form factors of the Graz potential. J. Phys. A, 22, 3597-3604. 
[30] Newton, R. G. (1982). Scattering Theory of Waves and Particles (Second edition). New York: Springer Verlag.

[31] Fuda, M. G., \& Whiting, J. S. (1973). Generalization of the jost function and its application to off-shell scattering. Phys. Rev. C, 8, 1255-1261.

[32] Laha, U. (2009). An integral transform of Green's function, off-shell Jost solution and T-matrix for coulomb-yamaguchi potential in coordinate representation. Pramana - J. Phys., 72, 457-472.

[33] Laha, U., \& Kundu, B. (2010). On the s-wave Jost solution for coulomb-distroted nuclear potential. Turkish J. Phys., 34, 149-157.

[34] Ghosh, D. K., Saha, S., Niyogi, K., \& Talukdar, B. (1983). Laplace transform method for off-shell scattering on nonlocal potentials. Czech. J Phys. B, 33, 528-539.

[35] Laha, U., \& Bhoi, J. (2013). Integral transform of the coulomb green's function by the Hankel function and off-shell scattering. Phys. Rev. C., 88, 064001(1-10).

[36] Laha, U., \& Bhoi, J. (2013). An integral transform of Coulomb Green's function via sturmian representation and off-shell scattering. Few-Body Syst., 54, 1973-1985.

[37] Sett. G. C., Laha, U., \& Talukdar, B. (1988). Phase-function method for coulomb-distorted nuclear scattering. J. Phys. A: Math. Gen., 21, 3643-3657.

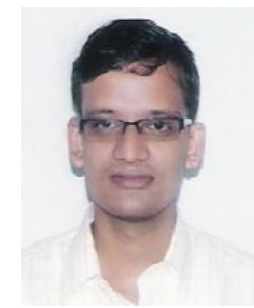

Jhasaketan Bhoi was born on $4^{\text {th }}$ May, 1981 in Talsrigida, Odisha, India. He has done his M. Sc. and MPhil degrees from Sambalpur University, Jyoti-Vihar, Burla, Sambalpur, Odisha, India with nuclear physics specialization. His major fields of interest include nuclear scattering theory, supersymmetry quantum mechanics and mathematical physics. He has published ten research papers in different journals of international reputation. Some of his recent publications are Integral transform of the Coulomb Green's function by the Hankel function and off-shell scattering in Phys. Rev. C. in 2013.

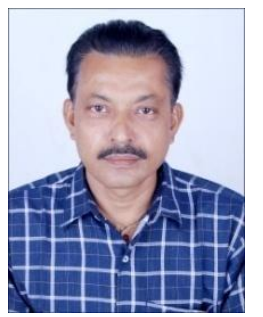

Ujjwal Laha was born on $4^{\text {th }}$ Feb., 1962 in Bholpur, Santiniketan, West-Bengal, India. He has obtained his Ph. D. degree from Visva-Bharati University, Santiniketan, West-Bengal, India. His areas of research interests include scattering theory, mathematical physics and supersummetry quantum mechanics. He has published more than sixty number of research papers in various journals of international reputations. Some of his recent publications include Comparative study of the energy dependent and independent two-nucleon interactions-a supersymmetric approach in International Journal of Modern Physics in 2014 etc. 